\title{
Topical tazarotene cream $(0.1 \%)$ in the treatment of facial acne: An open clinical trial
}

\author{
ASM Zakaria $^{1}$, HK Paul ${ }^{1}$, MA Rahman ${ }^{2}$, MT Islam³ AM Choudhury $^{1}$ \\ ${ }^{l}$ Department of Dermatology \& Venereology, Bangabandhu Sheikh Mujib Medical University, Dhaka; \\ ${ }^{2}$ Department of Respiratory Medicine, Bangabandhu Sheikh Mujib Medical University, Dhaka; \\ ${ }^{3}$ Consultant Dermatology, Gowainghat Upazilla Health Complex, Sylhet
}

\begin{abstract}
Background: Tazarotene is a new $3^{\text {rd }}$ generation topical acetylenic retinoid. It normalizes keratinocyte differentiation, reduces keratinocyte proliferation and decreases expression of inflammatory markers. Tazarotene was approved by US FDA in 1997 for acne vulgaris. Objectives: To evaluate the efficacy and safety of topical tazarotene $0.1 \%$ cream in the treatment of facial acne. Materials and methods: 67 patients with facial acne in the age range of 13-30 years were enrolled in the study. Purposive sampling was done. Patients were treated with topical Tazarotene cream $(0.1 \%)$ and were instructed to apply the medication as a thin film over the affected area in the evening once daily for 12 weeks. Follow-up was done at $2^{\text {nd }}, 4^{\text {th }}$ week, $8^{\text {th }}$ week and at $12^{\text {th }}$ week. Results: Of the 67 patients, $53 \%$ got remission, $9 \%$ had good response, $34 \%$ had poor response and there was no response in $4 \%$ of the patients by 12 weeks of treatment. Among the patients, 9 (13.43\%) developed mild side effects. Conclusion: Topical tazarotene cream $(0.1 \%)$ is a effective and safe treatment option for acne vulgaris affecting face. It is mostly effective in grade- 1 and grade- 2 acne.
\end{abstract}

\section{Introduction}

Acne vulgaris is a chronic inflammatory disease of the pilosebaceous follicles, characterized by comedones, papules, pustules, nodules, and cysts. It affects the areas of skin with the densest population of sebaceous follicles; these areas include the face, neck, the upper part of the chest, and the back, upper $\operatorname{arm}^{1}$.

Ninety percent of all teenagers may be affected with acne to some degree. It may begin in the twenties or thirties. As a rule, there is involution of the disease before age $25^{2}$. It can persist throughout adulthood in $12 \%$ of women older than 25 years and $3 \%$ of persons aged 35 to 44 years $^{3}$. Although not life-threatening acne markedly influences quality of life and constitutes a socioeconomic problem. Not less than $15-30 \%$ of acne patients require medical treatment due to the severity of their clinical condition ${ }^{4}$.

Various regimens and treatment modalities have been tried with varying degree of success. Neither of the available oral therapy (antibiotics and retinoids) nor the topical therapy is being considered as an ideal and fruitful treatment of acne vulgaris ${ }^{2}$. Tazarotene is a new $3^{\text {rd }}$ generation topical acetylenic retinoid. It normalizes keratinocyte differentiation, reduces keratinocyte proliferation and decreases expression of inflammatory markers. The selectivity of tazarotene for the $\beta$ and $\gamma$ subtypes of retinoic acid receptors minimizes the risk of adverse effects. Tazarotene has low potential for systemic adverse effects due to minimal absorption, its rapid metabolism into hydrophilic metabolites and rapid elimination from the body. There is no systemic accumulation of the drug. Tazarotene was approved by US FDA in 1997 for acne vulgaris ${ }^{5,6}$.

The present study will evaluate the efficacy and safety of tazarotene in Bangladeshi patients with acne vulgaris.

\section{Methodology}

This was a prospective open clinical trial. 74 consecutive patients of acne localized on the face attending the out-patient and in-patient dept. of Dermatology \& Venereology, Bangabandhu Sheikh Mujib Medical University, Shahbag, Dhaka was included in the study. Purposive sampling was done. Prior to enrollment written informed consent was taken.

All of the patients were in good health and did not receive any oral or topical anti-acne therapy in the 8 weeks prior to the study. Patients of both sexes in 
the age group of 13-30 years with the clinical diagnosis of facial acne of any grade from grade- 1 to grade-4 were enrolled. Pregnant and lactating women; acne conglobata and acne fulminans; patients with Known hypersensitivity to tazarotene; patients of hepatic or renal diseases were excluded.

Patients were treated with Tazarotene cream $(0.1 \%)$ and were instructed to apply the medication as a thin film over the affected area in the evening once daily for 12 weeks. Before treatment age, sex, occupation, socioeconomic status and type, site, grade, duration of acne lesion and previous treatment of the patients were recorded. The grading system used during dermatological examination to grade the acne are as follows: grade $-0=$ no visible lesion (remission), grade$1=$ comedones, papules and or pustules up to 5, grade- $2=$ comedones, papules and or pustules more than 5 , grade- $3=$ comedones, papules and or pustules (any number) plus single nodule/cyst, grade- $4=$ comedones, papules and or pustules (any number) plus multiple nodules/cysts. Duration of treatment was 12 weeks. Follow-up were done at $2^{\text {nd }}, 4^{\text {th }}, 8^{\text {th }}$ and $12^{\text {th }}$ week.

In each follow-up visit clinical evaluation was done counting inflammatory lesions and noninflammatory lesions present on the face. Efficacy was assessed by grading system mentioned above. Any patient who attains grade- 0 was considered as complete response or remission; improvement by 2 -grade that is from grade- 3 to grade- 1 or from grade-4 to grade-2 was considered as good response; improvement by 1-grade was considered as poor response; no improvement was considered as no response. The clinical safety was monitored by the incidence of treatment-emergent adverse events. Any adverse effects like burning, pruritus, erythema and dryness was noted. Each sign and symptom was rated on a $0-3$ scale, corresponding to none, mild, moderate or severe, respectively. A structured pretested questionnaire was used to collect information on demographic and clinical variables, treatment schedule, treatment outcome and the adverse events developed during the treatment.

At the end of the study, results were evaluated in 67 patients. Seven patients did not complete follow up.

\section{Results}

Out of all patients, $53.73 \%$ were within 16 to 20 years age group followed by $34.82 \%$ within 21 to 25 years, $6.0 \%$ above 25 years and $5.9 \%$ below 15 years age group. Mean age $( \pm$ SD) of the respondents was $20.33 \pm(3.2)$ years. All respondents were within 13 to 30 years age range. $32.8 \%$ were male and $67.2 \%$ were female. $80.6 \%$ were student followed by $8.9 \%$ were housewife, and $10.44 \%$ were engaged in other occupations.

Highest number of patients $(49.3 \%)$ was of lower middle class followed by $40.3 \%$ of upper middle class, $3.0 \%$ of upper class and $7.5 \%$ patients were poor (Table-I).

At baseline $23.9 \%$ had grade-1, $49.3 \%$ had grade-2, $17.9 \%$ had grade- 3 and $9.0 \%$ had grade- 4 acne. Mean duration \pm SD of acne of were $45.28 \pm(5.20)$ months (Table-II).

All the patients with grade- 1 acne, $16(100 \%)$ were in remission after the treatment period; 18 (54.5\%) of patients of grade-II were in remission and 15 (45.5\%) improved to grade-I (Poor response); 1 $(8.3 \%)$ of patients of grade-III were in remission, 5 (41.7\%) improved to grade-I (Good response) and $6(50.0 \%)$ improved to grade-II (Poor response); 1 $(16.7 \%)$ of patients of grade-IV were improved to grade-II (Good response), 2 (33.3\%) to grade-III (Poor response) and $3(50.0 \%)$ had no response (Table-III).

Out of 67 patients 53\% got remission, 9\% showed good response, $34 \%$ showed poor response and there were no response in $4 \%$ of the patients (Figure-1).

Among the patients $9(13.43 \%)$ developed side effects. Some of the patients developed multiple side effects. Of which $6(8.96 \%)$ had burning, 5 (7.46\%) had pruritus, 5 (7.46) had erythema, 7 (10.44) had scale and 8(11.94) had dryness of facial skin (Table-IV \& Figure-2).

\begin{tabular}{ll} 
Table-I: Socio-demographic variables & \\
\hline & Frequency (Percent) \\
\hline Age & \\
$<15$ & $4(5.9)$ \\
$16-20$ & $36(53.73)$ \\
$21-25$ & $23(34.82)$ \\
$>25$ & $4(5.9)$ \\
Sex & \\
Male & $22(32.8)$ \\
Female & $45(67.2)$ \\
Occupation & \\
Student & $54(80.6)$ \\
Housewife & $6(8.9)$ \\
Others & $7(10.44)$ \\
Socio-economic condition & \\
poor & $5(7.5)$ \\
Lower middle class & $33(49.3)$ \\
upper middle class & $27(40.3)$ \\
Upper class & $2(3.0)$ \\
\hline
\end{tabular}

Table-II: Distribution of the patients by grade of acne at base line.

\begin{tabular}{ll}
\hline Grade & Number (Percent) \\
\hline Grade 1 & $16(23.9)$ \\
Grade 2 & $33(49.3)$ \\
Grade 3 & $12(17.9)$ \\
Grade 4 & $6(9.0)$ \\
\hline Total & $67(100.0)$ \\
\hline
\end{tabular}


Table-III: Distribution of patients by response to treatment

\begin{tabular}{|c|c|c|c|c|c|c|}
\hline \multirow[t]{2}{*}{ Base line } & \multicolumn{5}{|c|}{ After Treatment } & \multirow[t]{2}{*}{ Total } \\
\hline & Remission & Grade-I & Grade-II & Grade-III & Grade-IV & \\
\hline Grade-I & $16(100.0)$ & $0(0.0)$ & $0(0.0)$ & $0(0.0)$ & $0(0.0)$ & $16(100.0)$ \\
\hline Grade-II & $18(54.5)$ & $15(45.5)$ & $0(0.0)$ & $0(0.0)$ & $0(0.0)$ & $33(100.0)$ \\
\hline Grade-III & $1(8.3)$ & $5(41.7)$ & $6(50.0)$ & $0(0.0)$ & $0(0.0)$ & $12(100.0)$ \\
\hline Grade-IV & $0(0.0)$ & $0(0.0)$ & $1(16.7)$ & $2(33.3)$ & $3(50.0)$ & $6(100.0)$ \\
\hline Total & $35(52.2)$ & $20(29.9)$ & $9(13.4)$ & $2(3.0)$ & $1(1.5)$ & $67(100.0)$ \\
\hline
\end{tabular}

Table-IV: Distribution of patients by side effects $(n=9 ; 13.43 \%)$ (multiple response)

\begin{tabular}{lc}
\hline Side effects & Number (Percent) \\
\hline Burning & $6(8.96)$ \\
Pruritus & $5(7.46)$ \\
Dryness & $8(11.94)$ \\
Erythema & $5(7.46)$ \\
Scaling & $7(10.44)$ \\
\hline
\end{tabular}

Figure-1: Pie-chart showing overall response to treatment.

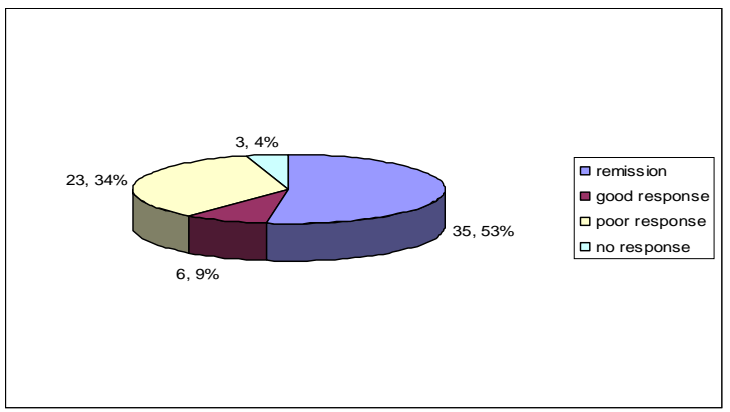

Figure-2: Doughnut showing treatment emergent side effects $(n=9)$

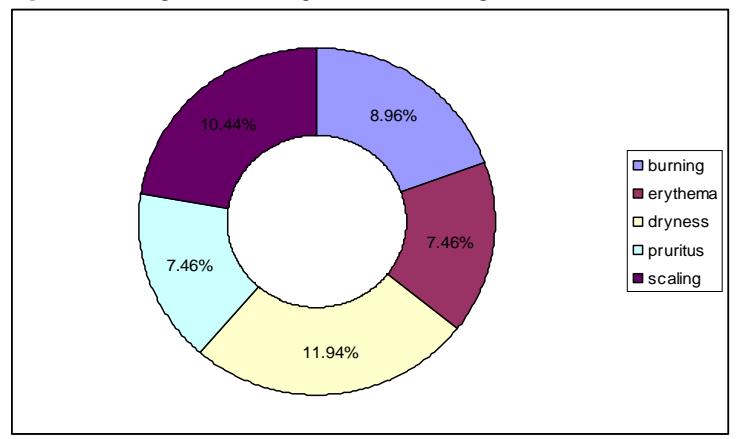

\section{Discussion}

Acne vulgaris is one of the most common disorders treated by dermatologists. Obstruction of the sebaceous follicle is the principal pathologic event, with formation of a microcomedo, the precursor of all acne lesions. When this obstruction occurs, the continued production of sebum and keratin gives rise to visible non-inflammatory lesions, open comedones (blackheads) and closed comedones (whiteheads). Leakage or rupture of the contents of the comedones into the dermis causes inflammatory acne $^{7}$.- The use of many of the available topical therapies is limited by inadequate long term efficacy, adverse effects and/or lack of cosmetic acceptability.

Tazarotene is a new third generation topical acetylenic retinoid. It normalizes keratinocyte differentiation, reduces keratinocyte proliferation and decreases expression of inflammatory markers. The selectivity of tazarotene for the beta and gamma subtypes of retinoic acid receptors minimizes the risk of adverse effects ${ }^{5,6}$.

At baseline $23.9 \%$ had grade-1, 49.3\% had grade-2, $17.9 \%$ had grade- 3 and $9.0 \%$ had grade- 4 acne.

Gradual declining trend of grade 4, 3, and 2 was observed in patients. After the treatment period of 12 weeks, all the patients with grade- 1 acne were in remission; $54.5 \%$ of patients of grade-II were in remission and $45.5 \%$ improved to grade-I (Poor response); $8.3 \%$ of patients of grade-III were in remission, $41.7 \%$ improved to grade-I (Good response) and $50.0 \%$ improved to grade-II (Poor response); $16.7 \%$ of patients of grade-IV were improved to grade-II (Good response), 33.3\% to grade-III (Poor response) and $350.0 \%$ had no response. It is evident that topical tazarotene cream $(0.1 \%)$ is more effective in grade- 1 and grade- 2 facial acne than that of their effect in grade- 3 and grade- 4 acne.

The efficacy of tazarotene in acne is estalished in studies conducted in India and in United states ${ }^{5,8,9}$. At the end of the $12^{\text {th }}$ week $93.6 \%$ of total study cases showed complete to moderate clearance of acne lesions ${ }^{5}$. There was a statistically significant reduction in both the inflammatory and noninflammatory lesion count at all study periods. The mean inflammatory acne count decreased by $84.5 \%$, and the non-inflammatory acne count decreased by $85.8 \%$ by 12 weeks ${ }^{8}$. Shalita et al. evaluated the safety and efficacy of topical tazarotene $0.1 \%$ and $0.05 \%$ gels. At week 12, treatment success rates were $68 \%$ and $51 \%$ for tazarotene $0.1 \%$ and $0.05 \%$, respectively ${ }^{9}$. Our results are comparable to these reports. 
Tazarotene has a low potential for systemic adverse effects due to minimal absorption, its rapid metabolism into hydrophilic metabolites and rapid elimination from the body. There is no systemic accumulation of the drug. Once-daily application leads to better patient compliance ${ }^{5,6}$.

Untoward effects were experienced by $11.9 \%$ of the patients during the treatment, most commonly itching $(4.8 \%)$, erythema and dryness $(2.4 \%)$ and burning $(1.6 \%)^{5}$. Adverse effects were noticed in 5 $(10.8 \%)$ patients, in the form of mild burning in 3 $(6.5 \%)$, itching in $3(6.5 \%)$, erythema in $2(4.3 \%)$ and desquamation in one $(2.2 \%)$ patient $^{8}$.

In our series, $9(13.43 \%)$ of the patients developed side effects, of which $6(8.96 \%)$ had burning, 5 (7.46\%) had pruritus, 5 (7.46) had erythema, 7 (10.44) had scale and 8 (11.94) had dryness of facial skin. Some of the patients developed multiple side effects. In our study side effects profile are slightly higher than that were reported by Saple DG et al. $^{5}$ and Nigam PK et $\mathrm{al}^{8}$. These may be due to over night application of the drug. Saple DG et al. and Nigam PK et al.used short contact therapy. All the adverse effects in our series were mild in nature. There was no study dropout because of adverse events.

In conclusion, over night application of topical tazarotene cream $(0.1 \%)$ might be an effective and safe treatment option for acne vulgaris affecting the face especially in grade- 1 and grade- 2 acne.

\section{References}

1. Harper JC. Acne vulgaris. emedicine. Last Updated: September 29, 2005 [Cited June 13, 2006]. Available from:URL:http://www.emedicine.com

2. Odom RB, James WD, Berger TG, editors. Andrews'. Diseases of the skin. Clinical dermatology, $9^{\text {th }}$ ed. Philadelphia: W. B. Saunders Company;2000

3. Thielitz A, Sidou F, Gollnick H. Control of microcomedone formation throughout a maintenance treatment with adapalene gel, $0.1 \%$. J Euro Acad Dermatol and Venereol 2007;21:747-53.

4. Zouboulis CC, Eady A, Philpott M, Goldsmith LA, Orfanos C, Cunliffe WC et al. What is the pathogenesis of acne? Exp Dermatol 2005;14:143-52.

5. Saple DG, Torsekar RG, Pawanarkar V, Dhanalakshmi UR,Ravichandran G, Kaur D et al. An open study to evaluate the efficacy and safety of tazarotene $(0.1 \%)$ in acne vulgaris.Indian $\mathrm{J}$ Dermatol Venereol Leprol 2004;70:92-95.

6. Foster RH, Brogden RN, Benfield P. Tazarotene. Drugs 1998;55:705-11.

7. Thiboutot D. Acne and rosacea: New and emerging therapies. Dermatol clin 2000;18:63-70

8. Nigam PK, Anant S. An evaluation of the efficacy and safety of tazarotene $(0.1 \%)$ cream in acne vulgaris. Indian J Dermatol Venereol Leprol 2005;71:360-1

9. Shalita AR, Chalker DK, Griffith RF et al. Tazarotene gel is safe and effective in the treatment of acne vulgaris: A multicenter, double-blind, vehiclecontrolled study. Cutis 1999;63:349-54 